\title{
Fabrication and nanoscale properties of PEDOT:PSS conducting polymer nanospheres
}

\author{
Matteo Sanviti ${ }^{1}$, Angel Alegria ${ }^{1,2, *}$, Daniel E. Martínez-Tong ${ }^{1,2, *}$ \\ ${ }^{1}$ Centro de Física de Materiales (CFM, CSIC-UPV/EHU). P. Manuel Lardizábal 5, 20018 San Sebastián - \\ Spain \\ ${ }^{2}$ Departamento Polímeros y Materiales Avanzados: Física, Química y Tecnología, University of the Basque \\ Country (UPV/EHU). P. Manuel Lardizábal 3, 20018 San Sebastián - Spain \\ *Corresponding authors: angel.alegria@ehu.eus
}

danielenrique.martinezt@ehu.eus

\begin{abstract}
Electrically conducting nanospheres of poly-(3,4-ethylenedioxythiophene):poly(styrene sulfonate) (PEDOT:PSS) with tailored size, were prepared by a one-step method. To fabricate the nanostructures, PEDOT:PSS was dissolved in ethylene glycol using a novel strategy and the solution was precipitated in deionized water. The proposed fabrication route allowed to obtain a water-based dispersion of monodisperse nanospheres with good optical properties. To determine physical properties of the nanospheres, we followed a nanoscale approach, using Atomic Force Microscopy (AFM). Our nanoscale mechanical and electrical investigations showed that the nanospheres preserved good physical properties, compared to the commercial product. Moreover, the local studies indicated that the confinement imposed by the spherical shape can lead into a different arrangement of the PSS and PEDOT phases. In particular, we envisaged nanospheres composed by a PEDOT-rich surface, responsible for the good electrical conductivity of the nanostructures.
\end{abstract}




\section{Introduction}

In the past decades, intrinsically conducting polymers have been an important research topic in the field of new and non-inorganic electronic devices, as alternatives to silicon-based technologies [1]. Among the reasons behind such great interest, we highlight the manufacturing versatility of polymers and their wide application window, which will lead soon to their use into the nanostructured technology landscape [2]. Nowadays, there are many different intrinsically conducting polymers commercially available [1]. Among them, poly(3,4-ethylenedioxythiophene):poly(styrenesulfonate) (PEDOT:PSS) is one of the most used and investigated [3] due to its high conductivity [4], transparency to visible light [5], and physicochemical stability [6]. For all these reasons, this material has found applications in organic solar cells [7], supercapacitors [8], organic light-emitting diodes [9], and sensors [10]. Commercial PEDOT:PSS is usually available as a water dispersion, composed by features with a usual size $\approx 25 \mathrm{~nm}$ in radius. The solid conductive polymer, obtained by casting the dispersion onto an appropriate substrate, has therefore a hierarchical structure, comprised by conducting grains connected by a PSS thin shell [11]. Moreover, the electric conductivity mechanism and the macroscopic properties of this material are strongly related to its nanostructured nature [12]. For this reason, the modification of the particles' dimensions and/or shapes could give to the material new bulk properties and extend the possible applications, especially in the field of nanostructured devices, where the conformation of the electrical contact at the nanoscale is fundamental [13].

The nanostructural engineering of conducting polymers has been widely studied for both fundamental research and potential applications [14]. In the particular case of PEDOT:PSS, it is crucial to have control over the superficial nanostructure of the polymer for most of its applications, since the hierarchical arrangement of PEDOT and PSS 
components dictate the resulting properties. Thus, several strategies had been adopted to develop a desired nano- or microstructured patterns, for example using electro-spinning [15], nanoimprint lithography (NIL) [16], and inkjet printing [17]. The increasing interest in finding reproducible and economic ways to tailor PEDOT:PSS nanostructures, has found recent interest in the scientific community, looking towards the use of advanced techniques and methodologies. In this context, Gutiérrez-Fernández et al. recently reported the nanopatterning of PEDOT:PSS thin films by laser induced periodic surface structures (LIPSS) [18]. The resulting nanograting consisted of electrically conducting ripples, opening a path to new possible applications of this material.

Moreover, in this area of research, intrinsically conducting and semiconducting polymeric nanospheres captured the attention of the scientific community in the past few years [19-21]. Recently, poly(3,4-ethylenedioxythiophene) (PEDOT) hollowed nanospheres were synthesized by Zhang et al. for applications into electrochromic systems [22]. The results showed that, for nanospheres with diameter around $100 \mathrm{~nm}$, the ion transport in the material was promoted, with respect to the denser and more compact bare PEDOT, underling the importance of tuning the dimensions of the particles for in PEDOT based system. This finding highlighted the importance that the control over the nanoparticle dimensions of the active layer has in systems such as supercapacitors. In the case of PEDOT:PSS, an important drawback for the fabrication of nanospheres is related to its bad solubility in most of commercial solvents with a low boiling point. To overcome these problems, several solutions, mainly involving its blends with other polymers, have been developed in order to give to the PEDOT:PSS better processability properties [2325]. However, this approximation comes with the limitation of introducing another polymer into the preparation process, which later on could be hard or even impossible to remove. Another option to dissolve this polymer is to exploit the PEDOT:PSS high 
affinity to polar high boiling point solvents, as ethylene glycol (EG) and dimethyl sulfoxide (DMSO) [26]. For example, the interactions between PEDOT:PSS and EG, results in a new molecular conformation of PEDOT and PSS, imposed by the solvent molecules after specific treatments [27-28]. This fact has led to a sensitive enhancement of the intrinsic conductivity of the polymer [29-30]. Thanks to such polymer/solvent affinity, it would be possible to dissolve the PEDOT:PSS in EG, opening the possibility to manufacture nanostructures directly from solution.

In this work, we present a novel method to fabricate nanostructured PEDOT:PSS samples, composed by well-defined nanospheres. We show a new strategy to solubilize PEDOT:PSS in EG able to use the commercial material as precursor, without chemical modifications, for the preparation of nanostructures. We investigated the physical properties of the fabricated nanospheres in solution and solid state, and demonstrated that the nanostructured samples preserve the highly conductive character of PEDOT:PSS. Our work opens the possibility of tuning the shape of this material at the nanoscale, in order to find new possible applications.

\section{Experimental section}

Materials. PEDOT:PSS was bought from Sigma-Aldrich (product \#655201) and used as received. The sample consisted of a water dispersion of the polymer, at a 3.0-4.0 wt\%. Ethylene glycol (EG, anhydrous, 99.8\%, Sigma-Aldrich, product \#324558) and Milli-Q water were used without further purification. For dialysis we used a dialysis tubing cellulose membrane (Sigma-Aldrich, product \#D9402-100FT) with typical molecular weight cut-off of $\sim 14,000 \mathrm{~g} / \mathrm{mol}$.

PEDOT:PSS thin films were prepared by spin coating $(3000 \mathrm{rpm}, 120 \mathrm{~s})$ the commercial dispersion onto Indium Tin Oxide (ITO) substrates (Ossila, product \#S111). 
The ITO substrates were cleaned by ultrasonication in acetone for $15 \mathrm{~min}$, at room temperature, prior use. All the deposited samples were left in a vacuum chamber (pressure $<10^{-4}$ bar) for at least $24 \mathrm{~h}$ before measurements. PEDOT:PSS thin films of different thicknesses were obtained by diluting the commercial product using distilled water (from $\sim 30 \mathrm{mg} / \mathrm{mL}$ to $\sim 5 \mathrm{mg} / \mathrm{mL}$ ).

The PEDOT:PSS nanospheres were prepared following the reprecipitation method, as carefully detailed in Section 3.1. For solid-state studies, the obtained nanospheres were deposited also onto ITO substrates and dried in vacuum prior measurements.

Methods. Liquid dispersions were characterized by Dynamic Light Scattering (DLS) and Ultraviolet-visible (UV-vis) spectrometry. The DLS measurements were carried out at $25{ }^{\circ} \mathrm{C}$ in a Zetasizer Nano-ZS apparatus, using disposable glass cuvettes. UV-vis spectra were recorded on an Agilent 8453A apparatus using a quartz cuvette, at room conditions.

AFM measurements were carried to study the structural, mechanical and electrical properties of the samples. The AFM measurements were carried out in a Multimode microscope, controlled using Nanoscope V electronics (Bruker), and running Nanoscope 8.15 software (Build R3Sr8.103795). All measurements were carried out at room conditions $\left(50 \% \mathrm{RH}, 24^{\circ} \mathrm{C}\right)$. All the AFM images and spectra analysis was carried out using the Nanoscope Analysis 1.90 software (Bruker).

The structural and mechanical studies were conducted using the PeakForce-QNM method (PF-QNM). In this case, we used Tap300Al-G probes by BudgetSensors. For obtaining quantitative mechanical maps and force spectra, the system was calibrated following recent literature reports [31-33], summarized in the following lines. First, we obtained the cantilevers' spring constant using Sader's method [34]. In general, we found 
that the probes presented values in the $20-30 \mathrm{~N} / \mathrm{m}$ range. Second, we obtained the socalled "deflection sensitivity" by performing a linear force-distance curve against a sapphire standard provided by Bruker $(1 \mathrm{~Hz}, \mathrm{Z}-\mathrm{ramp}=200 \mathrm{~nm}$, trigger threshold $=0.2$ $\mathrm{V})$. We obtained a usual value of about $35 \mathrm{~nm} / \mathrm{V}( \pm 10 \%)$. This deflection sensitivity value was verified during PF-QNM operation, using the same sapphire sample (PeakForce frequency $=2 \mathrm{kHz}$, PeakForce amplitude $=100 \mathrm{~nm}$, PeakForce setpoint $=0.2 \mathrm{~V})$. The "Sync Distance" parameter of the equipment was also obtained at this point and fixed during the measurements of the PEDOT:PSS samples. Finally, the tip radius was obtained by the evaluation of a force-distance curve on a polystyrene thin film, with a nominal Young's modulus $\left(E_{\mathrm{Y}}\right)$ of $3 \mathrm{GPa}$. The typical tip radius value was $10 \mathrm{~nm}$. For all nanomechanical experiments on the PEDOT:PSS samples, we used a fixed PeakForce setpoint value of $20 \mathrm{nN}$. Force spectroscopy measurements were carried out at selected regions of the samples by performing linear force-separation curves $(1 \mathrm{~Hz}$, Z-ramp $=200$ $\mathrm{nm}$, trigger threshold $=20 \mathrm{nN}$ ). From the nanomechanical maps and force spectra the Young's modulus was calculated using a simplified Derjaguin-Müller-Toporov (DMT) model [35-36]:

$$
F-F_{\mathrm{ADH}}=\frac{4}{3} \frac{E_{\mathrm{Y}}}{\left(1-\vartheta^{2}\right)} \sqrt{R} \delta^{3 / 2}
$$

where $F$ is the applied force, $F_{\mathrm{ADH}}$ is the probe-sample adhesion force, $E_{\mathrm{Y}}$ the Young's modulus, $\vartheta$ the Poisson ratio (taken as 0.33 [37]), and $\delta$ the probe-sample indentation.

The electrical conductivity of the nanospheres was measured by local I-V curves, using the same AFM in the so-called TUNA workspace. In this case, the samples were also deposited on ITO substrates that were later on connected to the AFM sample holder using silver conductive paint (RS components, product \#189-3593). TUNA measurements were carried out using conductive probes (DDESP-FM-10, Bruker; SCM- 
PIT-V2, Bruker). In TUNA experiments it is required a precise control of the forces between probe and sample to perform a proper surface scanning without scratching, while at the same time allowing a good electrical contact. Then, we calibrated the probes to allow force control, in a similar way to the mechanical calibration. We obtained the cantilevers' spring constant by Sader's method $(\sim 3 \mathrm{~N} / \mathrm{m})$ and calibrated the deflection sensitivity against a Sapphire standard $(\sim 55 \mathrm{~nm} / \mathrm{V})$. In this way, for all the TUNA characterization we used a fixed deflection setpoint of $0.36 \mathrm{~V}$, equivalent to $\sim 54 \mathrm{nN}$ of normal force. Based on the samples' topography, we performed I-V curves on particular areas of the surface, using a point-and-shoot mode. In these cases, we used the same deflection setpoint as the one for imaging. We applied a DC voltage ramp to the sample $\left(S_{\mathrm{BIAS}}\right)$ in the $-10 \mathrm{mV} \leq S_{\mathrm{BIAS}} \leq 10 \mathrm{mV}$ range, at $1 \mathrm{~Hz}$ rate and collecting about 9000 points. The current passing through the sample and the AFM probe was detected by the current amplifier (Extended TUNA) at a $100 \mathrm{nA} / \mathrm{V}$ sensitivity. For each sample, the corresponding current-voltage curve (I-V curve) was acquired. We calculated the conductance of the films by determining the slope of the curves via a fitting to a linear function, and obtained the resistance as its reciprocal value. From the resistance measurements, we estimated the samples' conductivity $(\sigma)$ as: $\sigma=\frac{h}{R \cdot s}$, where $h$ is the sample's thickness, $R$ the resistance, and $s$ the tip-sample contact area. In our work, the contact area was calculated using the Hertz contact mechanics approximation as [38]: $s=$ $\pi \cdot\left(R_{\text {tip }} \cdot F / E_{\mathrm{Y}}\right)^{2 / 3}$, where $R_{\text {tip }}$ is the tip end radius $(40 \mathrm{~nm}$, according to the manufacturer), $F$ the applied force during I-V spectroscopy $(\sim 54 \mathrm{nN})$, and $E_{\mathrm{Y}}$ the calculated Young's modulus from the nanomechanical analysis (Table 1).

Surface electrical conductivity measurements were conducted on thin films with a four-point probe apparatus (Ossila, T2001A3), with a probe spacing of $1.27 \mathrm{~mm}$, a set current range of $20 \mathrm{~mA}$, and target current $5 \mathrm{~mA}$. 


\section{Results \& Discussion}

3.1. Fabrication of conducting polymer nanospheres. Scheme 1 presents the overall process of PEDOT:PSS nanospheres fabrication. First we started by analyzing the structure of the commercial PEDOT:PSS dispersion by DLS (Figure 1a, triangles). We observed that the sample was composed by nanometric structures of $\sim 50 \mathrm{~nm}$ in diameter (Z-average value), as previously reported [11].

(a) PEDOT:PSS solution in ethylene glycol

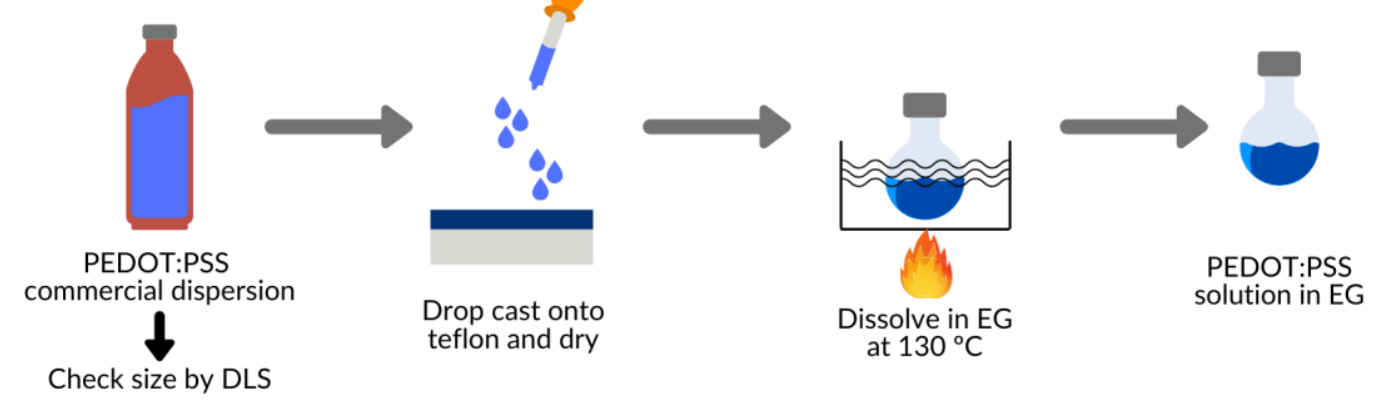

(b) PEDOT:PSS nanospheres by reprecipitation

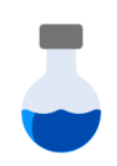

PEDOT:PSS

solution in EG

at room

temperature

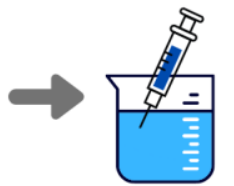

Fast injection in distilled wate
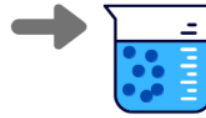

Dispersion of PEDOT:PSS nanospheres

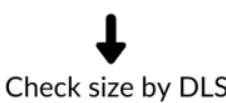

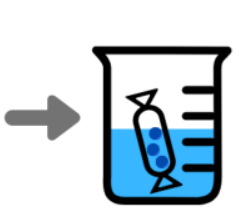

Dialysis against distilled water for $48 \mathrm{~h}$

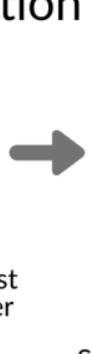

夏

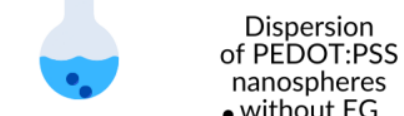

Syringe filter

nanospheres

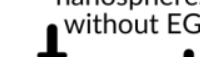

Check size by DLS optical properties by UV-Vis

(c) PEDOT:PSS nanospheres casting

\section{Sample 1}

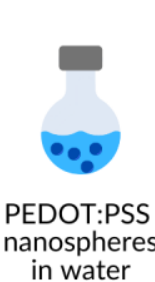

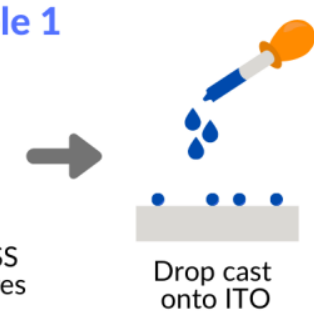

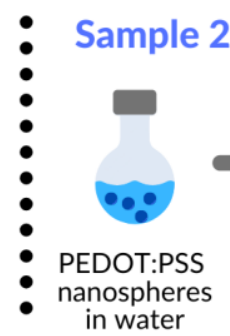

in water

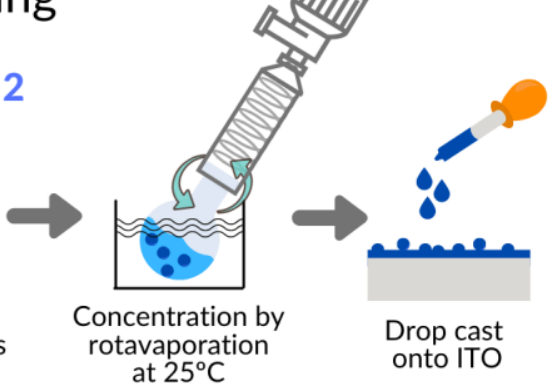

Nanoscale physical studies:

- Structural Mechanical - Electrical

Scheme 1. Scheme showing the PEDOT:PSS nanospheres' fabrication and studies. (a) Strategy to dissolve PEDOT:PSS in EG. (b) Fabrication of PEDOT:PSS nanospheres by reprecipitation. (c) Casting of PEDOT:PSS nanospheres for physical studies. 
In order to prepare the polymer nanospheres, we followed the so-called "reprecipitation method". This protocol has been widely employed in the literature to prepare polymer nanospheres of different chemical nature [39]. This method is a solventdisplacement technique that relies on crashing out hydrophobic polymer chains in solution by displacing a solvent with a non-solvent, generally water [40]. The polymer solvent and the non-solvent must be miscible with each other. First, we dried the asreceived PEDOT:PSS dispersion and redissolved it in EG (Scheme 1a). $100 \mu \mathrm{L}$ of the commercial PEDOT:PSS dispersion were drop-casted on a Teflon substrate. The dispersion was left in a vacuum oven (pressure $<10^{-1}$ bar) for 2 hours at room temperature to allow water evaporation. Then, the sample was transferred to a higher vacuum chamber (pressure $<10^{-4}$ bar) and left overnight $(\sim 16 \mathrm{~h})$. After complete drying, we obtained a dark blue homogeneous film. $1 \mathrm{mg}$ of solid PEDOT:PSS was cut from the dried film and dissolved in $1 \mathrm{~mL}$ of pure EG at $130^{\circ} \mathrm{C}$ under stirring for $\sim 3$ hours, resulting in a pale blue homogeneous solution. We highlight that after cooling down from $130{ }^{\circ} \mathrm{C}$ to room temperature $\left(\sim 25^{\circ} \mathrm{C}\right)$, the PEDOT:PSS in EG remained soluble, i.e., no macroscopic precipitation was observed.
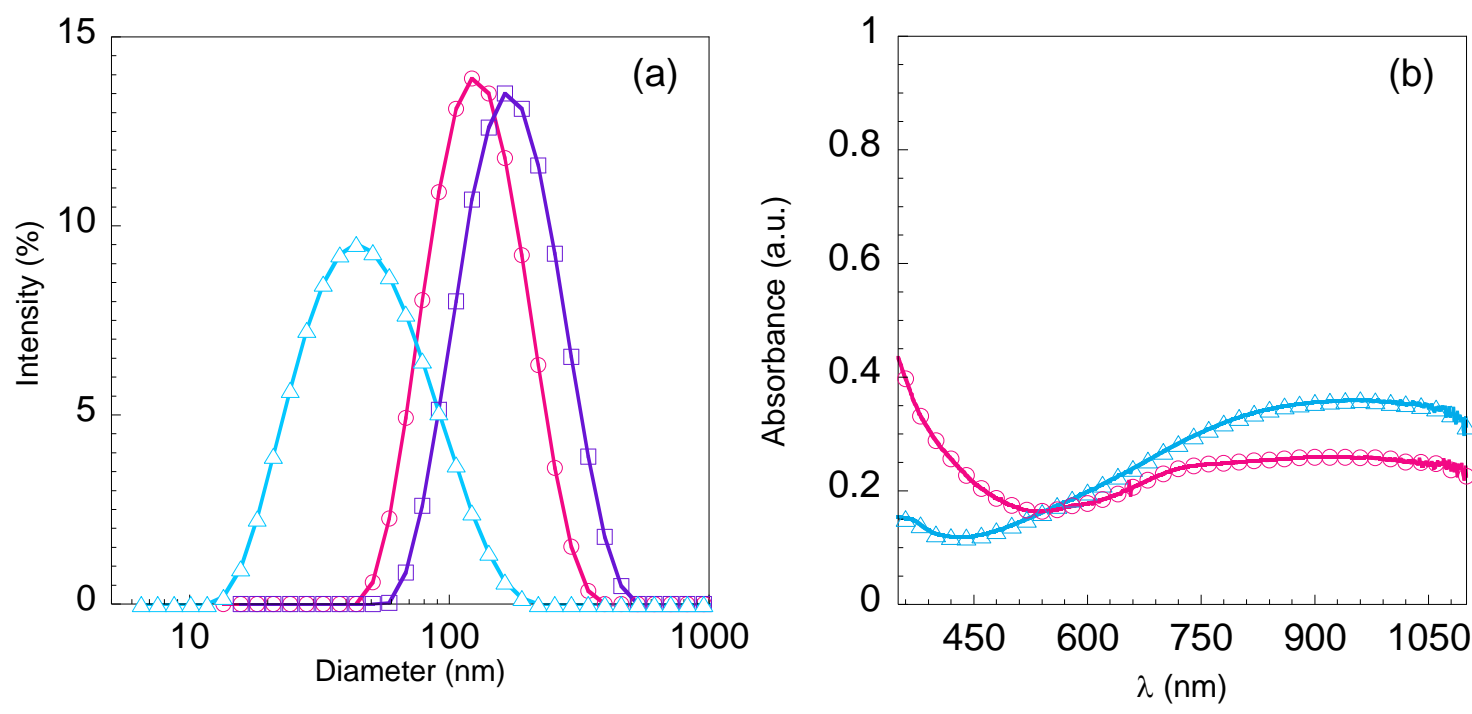
Figure 1. (a) DLS results as intensity as a function of sample diameter: commercial PEDOT:PSS (triangles), PEDOT:PSS nanospheres (squares), purified PEDOT:PSS nanospheres (circles). (b) UV-Vis results: commercial PEDOT:PSS (triangles) and the purified nanospheres (circles). The UV-Vis data is shown as 1 point of every 5 measurements.

For the nanospheres preparation, the PEDOT:PSS solution in EG was injected into $10 \mathrm{~mL}$ of $\mathrm{H}_{2} \mathrm{O}$ using a syringe, at room temperature and under stirring (Scheme $1 \mathrm{~b}$ ). Immediately afterwards, we performed a DLS measurement to check the PEDOT:PSS structure in the dispersion (Figure 1a, squares). The results showed that the reprecipitated PEDOT:PSS dispersion was composed of features reaching a Z-average value of $160 \mathrm{~nm}$ in diameter. This result represented an increase in about a factor 3 compared to the diameter of the as received PEDOT:PSS dispersion. Also, the width of the distribution was narrower for the reprecipitated sample, compared to the commercial PEDOT:PSS. In other words, the reprecipitation process allowed the fabrication of customizable nanostructures, different in size from the original dispersion with a better size dispersion.

The EG, still present in the aqueous dispersion, was separated from the nanospheres by dialysis against water. The dialysis process was carried out in a $2 \mathrm{~L} \mathrm{H}_{2} \mathrm{O}$ reservoir, with continuous water changes throughout 48 h. Finally, the resulting nanospheres dispersion was filtered using a PTFE filter $(0.2 \mu \mathrm{m})$. The DLS measurements of the obtained nanospheres are presented in Figure 1a (circles). The observed unimodal distribution of sizes had a Z-average value of $120 \mathrm{~nm}$ in diameter, indicating a small decrease when compared to the sample prior dialysis. This decrease could be related to the expel of residual EG, which was previously swelling the polymers. The width of the distribution did not show any particular changes, remaining narrower as compared to the commercial product. We will refer to the final product with the name of PEDOT:PSS nanospheres. 
Previous reports on polymeric nanospheres obtained by reprecipitation methods, showed that the manufacturing conditions have big impact over the dimensions, the internal structure and agglomeration tendency [41]. The just presented preparation method, was found to be the optimal case for obtaining monodisperse PEDOT:PSS nanospheres. Increasing the concentration of PEDOT:PSS in EG to, and above, $5 \mathrm{mg} / \mathrm{mL}$ resulted in an insoluble mixture. This fact hindered the possibility of preparing potentially bigger features, as previously reported [42]. Moreover, decreasing the concentration below $1 \mathrm{mg} / \mathrm{mL}$ resulted in a water dispersion comparable to the commercial PEDOT:PSS. We highlight that the $120 \mathrm{~nm}$ diameter of the obtained PEDOT:PSS nanospheres was comparable to those reported for conducting polymer nanospheres [41], for example based on poly(3-hexylthiophene) [20,43], PCDTBT [44], and poly(pphenylene ethynylene) [45].

In order to characterize the optical properties of the resulting nanospheres dispersion, we performed UV-Vis measurements. Figure $1 \mathrm{~b}$ shows the obtained spectra for the commercial PEDOT:PSS product (triangles) and nanospheres (circles). We observed that, after reprecipitation, the UV-Vis signal only suffered slight changes. For example, the characteristic bipolaron absorption band at around $800 \mathrm{~nm}$ was less pronounced in the nanospheres, compared to the as-received sample. This result was comparable to the one found for the synthesis of PEDOT:PSS using SCNPs [46] and enzymes [47]. Also, we noticed a lack of peaks around $\lambda=600 \mathrm{~nm}$, which indicated that the PEDOT chains preserved their oxidation state as previously reported by Heuer et al [48]. Finally, the UV-Vis signal of the PEDOT:PSS nanospheres for $\lambda>900 \mathrm{~nm}$ remained constant, also supporting the fact that the nanospheres fabrication process did not alter the chemical nature of the PEDOT:PSS complex [49-50]. 


\subsection{Film formation and solid-state properties of PEDOT:PSS nanospheres.}

The morphology and structure of dried PEDOT:PSS nanospheres were studied by AFM measurements. Figure 2 shows AFM topography images of the drop-casted nanospheres on ITO substrates, following two different procedures (Scheme 1c). First, Figure 3a shows a $5 \times 5 \mathrm{~m}^{2}$ topography image of a purified PEDOT:PSS nanospheres' dispersion in water, drop casted onto ITO (Sample 1). We observed that the dispersion was not concentrated enough to allow a continuous coating of the substrate, resulting in a distribution of single and isolated PEDOT:PSS nanospheres. The size of the nanostructures on the surface was around $140 \mathrm{~nm}$ in diameter with mean height of around $50 \mathrm{~nm}$, indicating that possible interaction with the surface during drying could affect the final morphology. The topography of the free surface surrounding the nanospheres matched the characteristics of ITO (mean roughness $\approx 3.8 \mathrm{~nm}[51]$ ), indicating that the nanospheres' dispersion did not contain any polymer residues able to form surface layers, at least by a usual AFM inspection. Second, Figure $2 \mathrm{~b}$ shows a $5 \times 5 \mu \mathrm{m}^{2}$ topography image of a concentrated nanospheres sample (Sample 2). In this case the mother dispersion of nanospheres was concentrated using a rotary evaporator $\left(T=25^{\circ} \mathrm{C}\right)$ starting with a 10 $\mathrm{mL}$ volume, until the water content decreased down to $\sim 0.5 \mathrm{~mL}$. After drop casting the concentrated solution, we observed a coating of the surface, characterized by the aggregation of the nanospheres in the solid state (Figure 2b). The top surface was not homogeneous, but composed of a distribution of spheres, resulting in different heights. The mean roughness of the sample surface was found to be $\sim 8 \mathrm{~nm}$. By scratching the resulting film, AFM measurements showed that the coating had a typical thickness of 50 nm. However, the range between maximum and minimum thickness varied from 35 to $350 \mathrm{~nm}$, meaning that the observed continuous coating was composed of aggregates with different dimensions. As a comparison, Figure 2c shows a PEDOT:PSS thin film 
deposited on ITO, using the commercial product. The spin casting resulted in a continuous thin film with a characteristic thickness of $\sim 430 \mathrm{~nm}$, as determined by AFM. This sample, labeled as Sample 0, showed the characteristic structure of PEDOT:PSS [52], comprised by stacking of small grains with a diameter of about $50 \mathrm{~nm}$, and an overall flatter surface $($ roughness $=3.5 \mathrm{~nm})$ compared to Sample 2 .
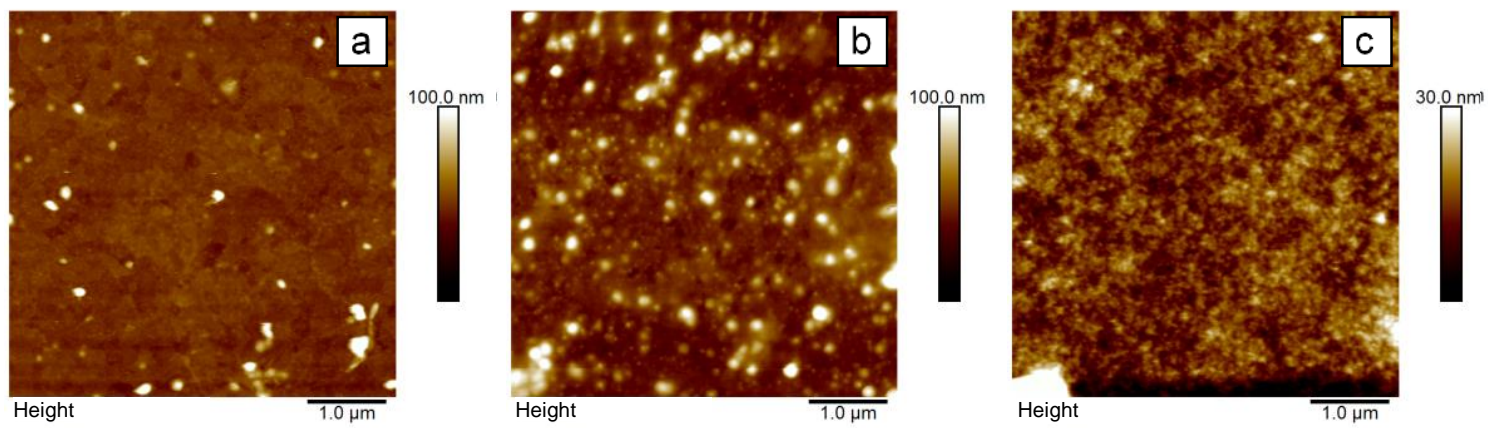

Figure 2. AFM topography (height) images of PEDOT:PSS nanospheres (a: Sample 1; b: Sample 2), and commercial PEDOT:PSS (c: Sample 0).

The evaluation of the dried PEDOT:PSS nanospheres' physical properties was carried out using AFM techniques. This approach allowed determining the individual response of each nanostructure. First, we studied the nanomechanical properties of Sample 0 to be used as reference for the interpretation of the nanospheres' results. 


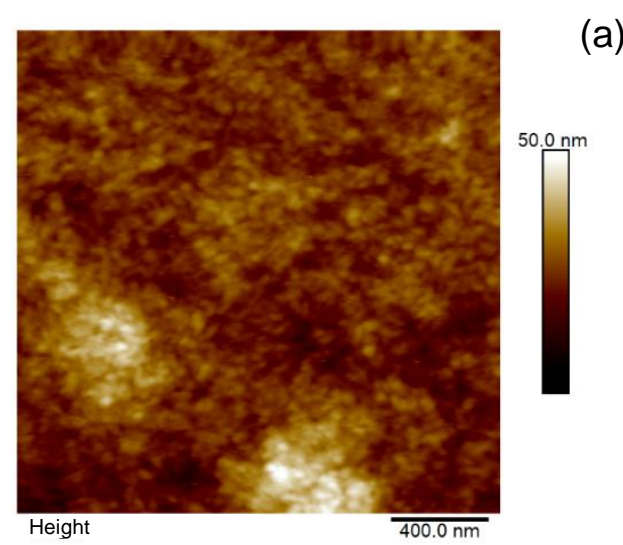

(a)

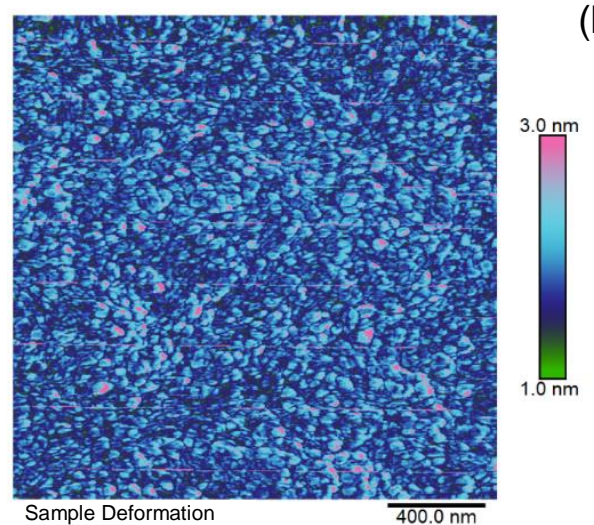

(b)

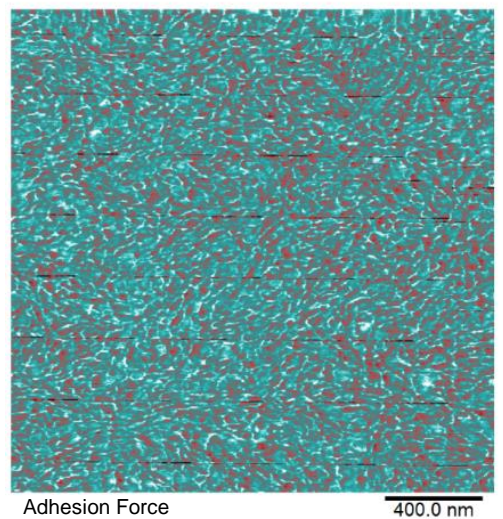

(c)

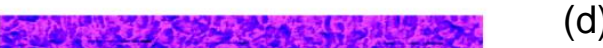

(d)

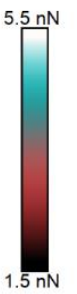

Modulus distribution over the acquired image
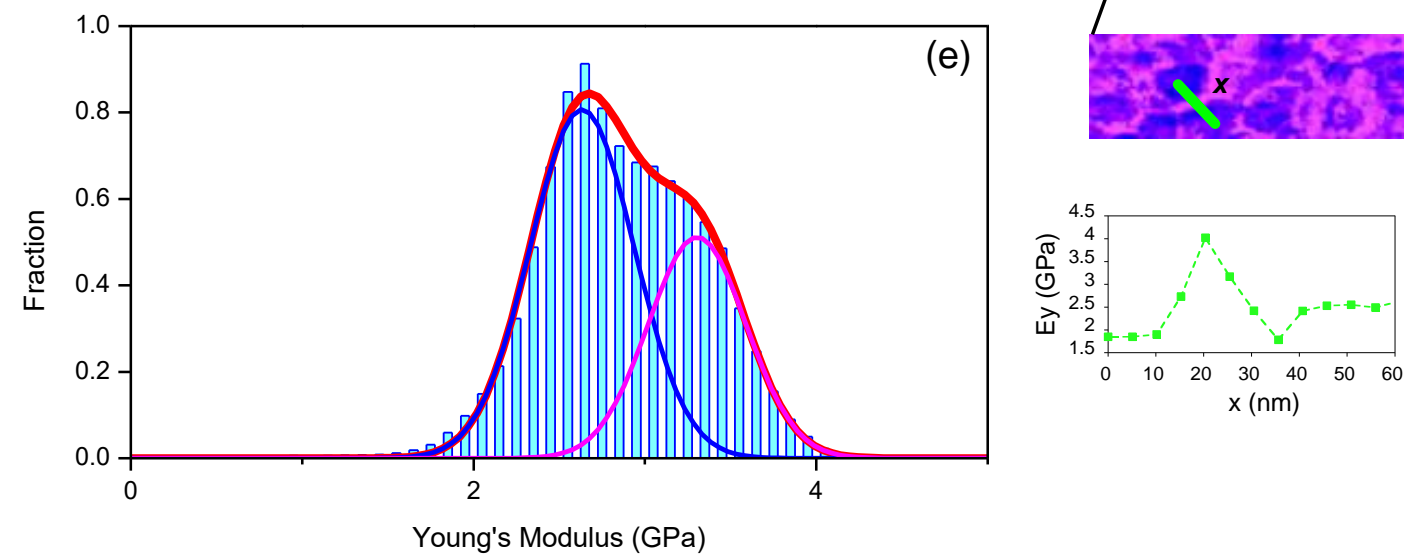

Figure 3. PF-QNM results for pristine PEDOT:PSS (Sample 0). (a) Topography, (b) deformation, (c) probe-sample adhesion force, and (d) Young's Modulus. (e) Distribution of Young's modulus. (f) Zoomin of the Young's modulus maps and cross-section, showing the different modulus contributions.

Figure 3 presents the nanomechanical results for Sample 0, using the PF-QNM technique. In the $2 \times 2 \mu \mathrm{m}^{2}$ topography image (Figure 3a) we observed the PEDOT:PSS 
grains with a high level of detail. The corresponding dynamic nanomechanical maps (obtained simultaneously) presented contrast (Figures 3b-d). To quantify the mechanical response associated with this contrast, Figure $3 \mathrm{e}$ presents a histogram of Young's modulus values, calculated using the map presented in Figure 3d. The histogram showed a non-monotonous distribution, characterized by a maximum around $2.5 \mathrm{GPa}$, plus a broad shoulder towards higher modulus values. Comparing with the literature, the quantified Young's modulus range of Sample 0 was slightly lower, but in the same order of magnitude, respect to that reported by Gutiérrez-Fernández et al. for an inkjet-printed thin film of comparable thickness, also using PF-QNM [53].

In order to further analyze this result, the histogram was fitted by a sum of two Gaussian functions (fitting curve $\mathrm{R}^{2}=0.99$, red line in Figure 3e). We can ascribe these functions to the representation of two distinct modulus populations (blue and fuchsia curves in Figure $3 \mathrm{e})$. The mean value $(\mu)$ for these populations was $\mu_{1}=2.6 \mathrm{GPa}$ and $\mu_{2}$ $=3.3 \mathrm{GPa}$, respectively, with comparable standard deviations of $\sim 0.4$. These populations can be related to local mechanical differences between the PEDOT:PSS rich grains and a PSS interconnecting layer. A zoom-in of the Young's modulus map showed that the grains were the ones presenting the lower modulus, while the interconnecting layer was characterized by the higher modulus (Figure 3f). These findings were in line with previous reports, where it was shown that the PSS rich phase in PEDOT:PSS was characterized by higher modulus than the granules constituting the material [54]. Moreover, in a recent report by Qu et al., the authors quantified a Young's modulus of about $\sim 2.5 \mathrm{GPa}$ for PEDOT thin films using PF-QNM [55]. This value is very similar to the one we report for the PEDOT:PSS rich grains. 
Finally, our PF-QNM nanomechanical studies showed that the PSS phase presented a lower sample deformation (Figure 3b), and a higher probe-sample adhesion force (Figure 3c), compared to the PEDOT:PSS grains. Also, from the mechanical maps we were able to calculate the width of the PSS interconnecting layer between the grains, resulting in a typical value of $\sim 15 \mathrm{~nm}$, while the typical diameter of the grains was $\sim 60$ nm. These results were in line with the expectations by comparing with results already reported in literature [11].

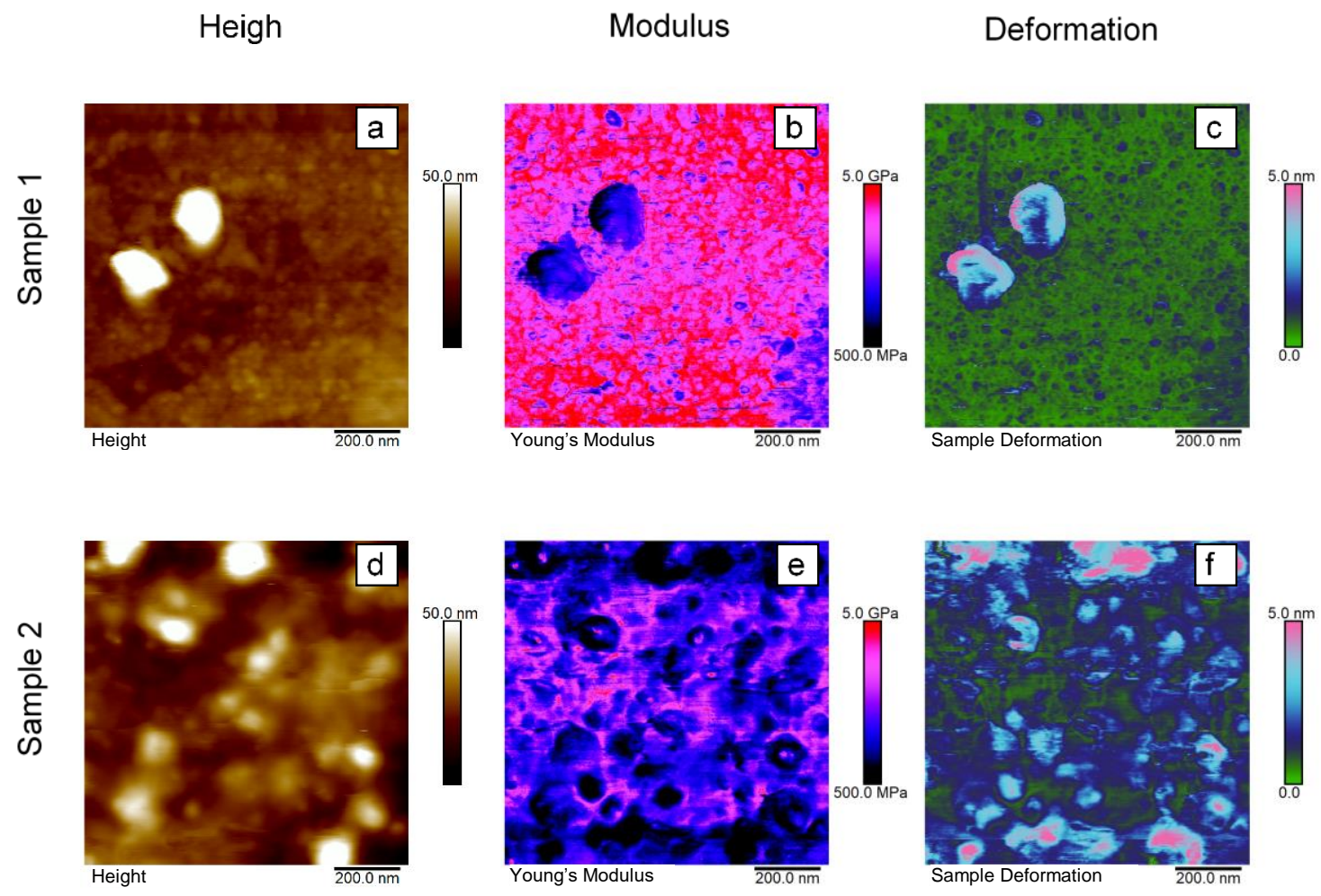

Figure 4. PF-QNM images and mechanical maps. Height (a,d); Young's modulus (b,e); Deformation (c,f).

Figure 4 shows $1 \mathrm{x} 1 \mu \mathrm{m}^{2}$ dynamic nanomechanical maps of PEDOT:PSS nanospheres. In particular, Figures 4a-c present the results for isolated spheres (Sample 1), Figures 4d-f for concentrated spheres (Sample 2). Our measurements showed that all 
the samples presented Young's modulus values in the GPa scale; however, in both cases, the nanospheres had a lower Young's modulus and lower sample deformation, compared to Sample 0. As reported recently [56], the geometry of polymer nanostructures might influence a proper quantification of mechanical properties by PF-QNM maps alone. Also, problems as topography crosstalk and the influence of the sinusoidal excitation of the piezo scanner during mapping [57] might affect a precise quantification of mechanical properties by PF-QNM maps. Then, to confirm possible mechanical changes in the PEDOT:PSS nanospheres, and enhance the nanoscale mechanical characterization, we performed force spectroscopy measurements on selected regions of the samples.
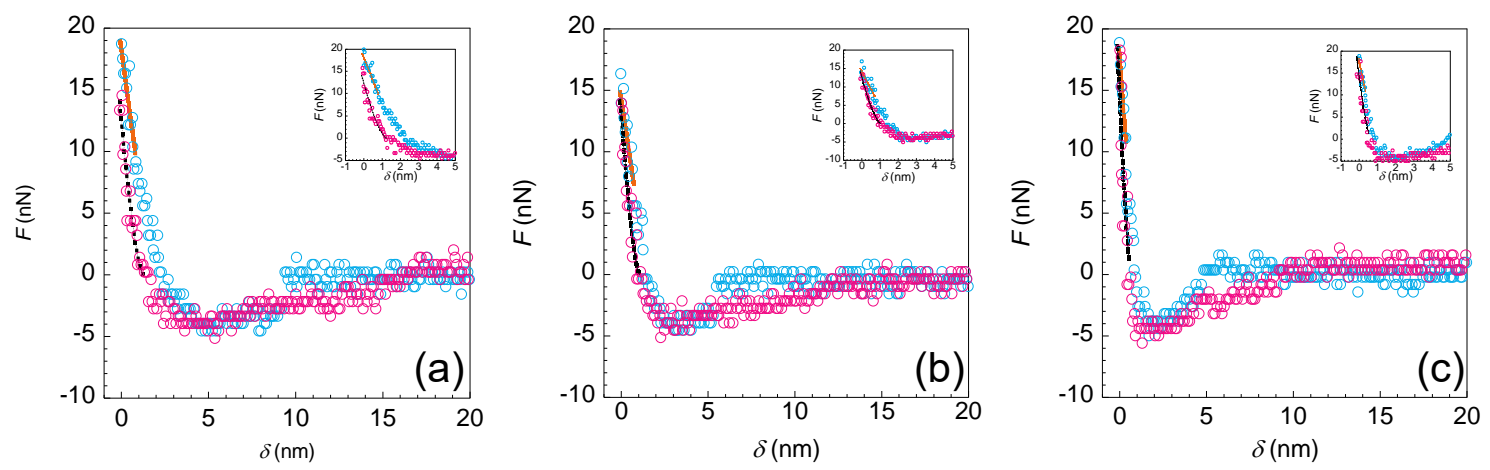

Figure 5. Force spectroscopy measurement results for Sample 1 (a), Sample 2 (b), and Sample 0 (c). The data is shown as 1 point every 5 measurements. Blue data points correspond to the probe-sample approach, while pink data points to the probe-sample retract. The continuous orange line shows the fitting for calculating the samples' stiffness, while the dashed black line presents the fitting to the Hertz model. In all frames, the inset shows the interaction range of the force spectroscopy measurements. In the insets the data is shown as 1 point every 3 measurements.

Figure 5 shows representative force-separation curves for each sample, obtained by the force spectroscopy technique. We note to the reader that in the case of the PEDOT:PSS nanospheres, the force-separation curves were captured on top of the nanospheres. This approximation aimed to avoid possible impact of nanostructure geometry on the mechanical properties' determination, as discussed in the literature [58]. The obtained results from this quasi-static approach are summarized in Table 1. The PEDOT:PSS nanospheres were softer compared to Sample 0, a result in line with the one 
obtained from the nanomechanical maps. The samples' stiffness was calculated from the force-separation curve as $\Delta F / \Delta \delta$, by fitting a linear function to the approach data (blue points in Figure 5), in the 50-90\% total force range. We quantified a stiffness decrease in the nanostructured samples of over $50 \%$, from $20 \mathrm{~N} / \mathrm{m}$ to $8 \mathrm{~N} / \mathrm{m}$, compared to the thin film. As expected, these results were accompanied by a higher indentation depth for the nanospheres, of about a factor 2. A size-dependent stiffness reduction was also observed in a previous report comparing PEDOT:PSS films at different thicknesses, using AFMbased contact resonance measurements [59]. The authors suggested that the softening was related to a different structural arrangement in the thinner samples.

The Young's modulus, also representing a measure of the samples' stiffness, was calculated by fitting eqn (1) to the retract data (Figure 5), in the $10-90 \%$ total force range. We observed a decrease of the mean $E_{\mathrm{Y}}$ from $3.4 \mathrm{GPa}$ to $2.1 \mathrm{GPa}$, comparing Sample 0 with the PEDOT:PSS nanospheres. These results confirmed the previous observations by PF-QNM using Young's modulus maps. Considering that PEDOT has a lower $E_{\mathrm{Y}}$ compared to PSS [54,60], the quantified softening of the nanospheres could be related to a different distribution of PEDOT and PSS in the resulting nanospheres after reprecipitation. This idea is in line with a possible re-arrangement of the PEDOT and PSS phases in confined geometries, as introduced in the last paragraph. Then, in this scenario, the modulus decrease would indicate a PEDOT enriched outer shell in the prepared nanospheres. This idea is also in line with previous reports of PEDOT:PSS ultra-thin films (thickness $<100 \mathrm{~nm}$ ), where Young's modulus values ranging between 1 - 2 GPa [53,61] were reported.

Table 1. Quantitative nanomechanical data from force spectroscopy measurements. $E_{\mathrm{Y}}$ indicates the Young's modulus, and $F_{\mathrm{ADH}}$ the adhesion force. The last column of the table shows the number of force curves analyzed for each sample. For nanospheres, the force curves were taken at the top of the sphere. 


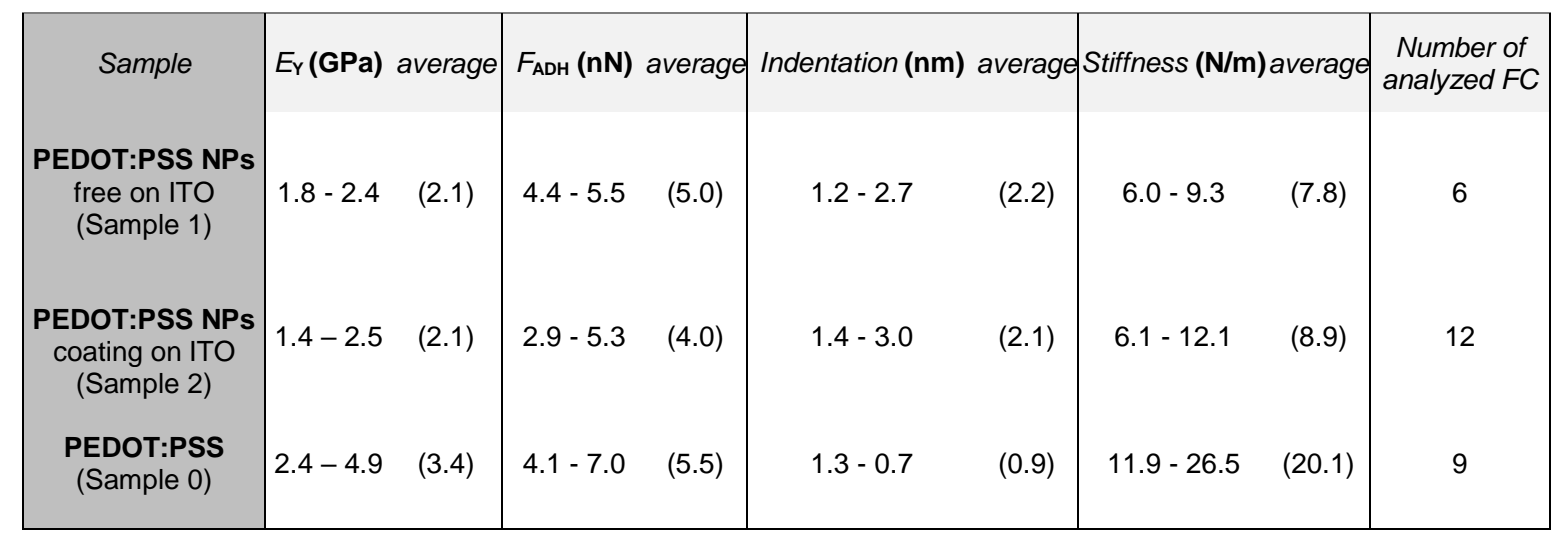

Finally, we explored the functionality of the PEDOT:PSS nanospheres by studying their electrical conductivity at the nanoscale, by TUNA experiments. Figure 6a shows the PEDOT:PSS topography, as determined by TUNA (contact mode experiment). No apparent damage to the surface was performed during scanning. Figure $6 \mathrm{~b}$ presents a characteristic I-V curve obtained for this sample, at the position indicated in the topography image. The I-V curve showed an Ohmnic response, from which the conductivity was calculated as the slope of the line. We found a mean conductivity $(\sigma)$ of $90 \mathrm{~S} / \mathrm{cm}$ for Sample 0. This value was obtained as the average over 5 measurements on different positions of the sample (Table 2). We highlight that the TUNA conductivity measurements resulted in a broad distribution of conductivity values, which varied depending on the probed area. However, all the results were within the same order of magnitude. These local differences can be related to small changes in the PEDOT:PSS effective concentration at each probed point. Also, we emphasize that, considering the experimental setup and sample preparation procedure, the TUNA conductivity measurements must account for an out-of-plane conductivity of the sample. In order to complement the results, we measured the in-plane conductivity of Sample 0 by the fourpoint probe technique. We found an in-plane conductivity of $3.6 \cdot 10^{2} \mathrm{~S} / \mathrm{cm}$; a value in line with the description of the product provider $(\sigma>200 \mathrm{~S} / \mathrm{cm})$, and comparable to those 
reported for different PEDOT:PSS thin films in the past years [61-63]. The observed differences between in-plane and out-of-plane conductivity response of PEDOT:PSS were in line with a previously report by Nardes et al [64]. In that work, the authors explained that these differences were related to an anisotropy in the disposition of the PEDOT:PSS grains during film formation, where the conducting granules would tend to preferentially arrange coaxially and perpendicular to the substrate plane.

Table 2. Conductance and conductivity values of PEDOT:PSS thin films for different thicknesses.

\begin{tabular}{|c|c|c|}
\hline Thickness (nm) & Conductance $(1 / \mathrm{M} \Omega)$ & Conductivity $(\mathrm{S} / \mathrm{cm})$ \\
\hline 430 & $9.4 \pm 7$ & $(9.0 \pm 6) \cdot 10^{1}$ \\
\hline 225 & $9.0 \pm 7$ & $(5 \pm 3) \cdot 10^{1}$ \\
\hline $\begin{array}{c}75 \\
\text { Nanosphere } \\
55\end{array}$ & $\begin{array}{c}1.1 \\
4.8 \pm 3\end{array}$ & $\begin{array}{c}2 \\
6 \pm 4\end{array}$ \\
\hline $\begin{array}{c}50 \\
\text { Nanosphere } \\
20\end{array}$ & $\begin{array}{c}1.6 \cdot 10^{-1} \\
0.66 \pm 0.60\end{array}$ & $\begin{array}{c}0.2 \\
0.3 \pm 0.2\end{array}$ \\
\hline
\end{tabular}

In the case of PEDOT:PSS nanospheres, we also determined the nanoscale conductivity of the nanostructures by TUNA experiments. We clarify to the reader that these experiments were not possible to be properly conducted on Sample 1, due to the following technical limitation. Since the experiments required scanning in AFM contact mode, we observed that during scanning the isolated nanospheres were swept away by the probe. When extremely small forces $(<<1 \mathrm{nN})$ were used, contact mode scanning was possible without sweeping the structures. However, the probe-sample electrical contact was not adequate to guarantee a proper conductivity measurement. However, we were able to study the electrical properties of Sample 2 (Figures $6 \mathrm{c}, \mathrm{d}$ ).

Figure $6 \mathrm{c}$ shows the contact mode height images of Sample 2. The obtained topographical map was comparable to those already presented in this work, indicating no important damage of the probe while contact mode imaging. Figure $6 \mathrm{~d}$ shows the I-V 
response of PEDOT:PSS nanospheres at two different probed areas. In both cases, the data showed a linear trend, indicating that the nanospheres presented an Ohmnic conductivity behavior. The I-V analysis resulted in conductivities of 2.0 and $0.2 \mathrm{~S} / \mathrm{cm}$, depending on the probed area; as the measurement point was higher in topography, the higher the obtained conductivity.
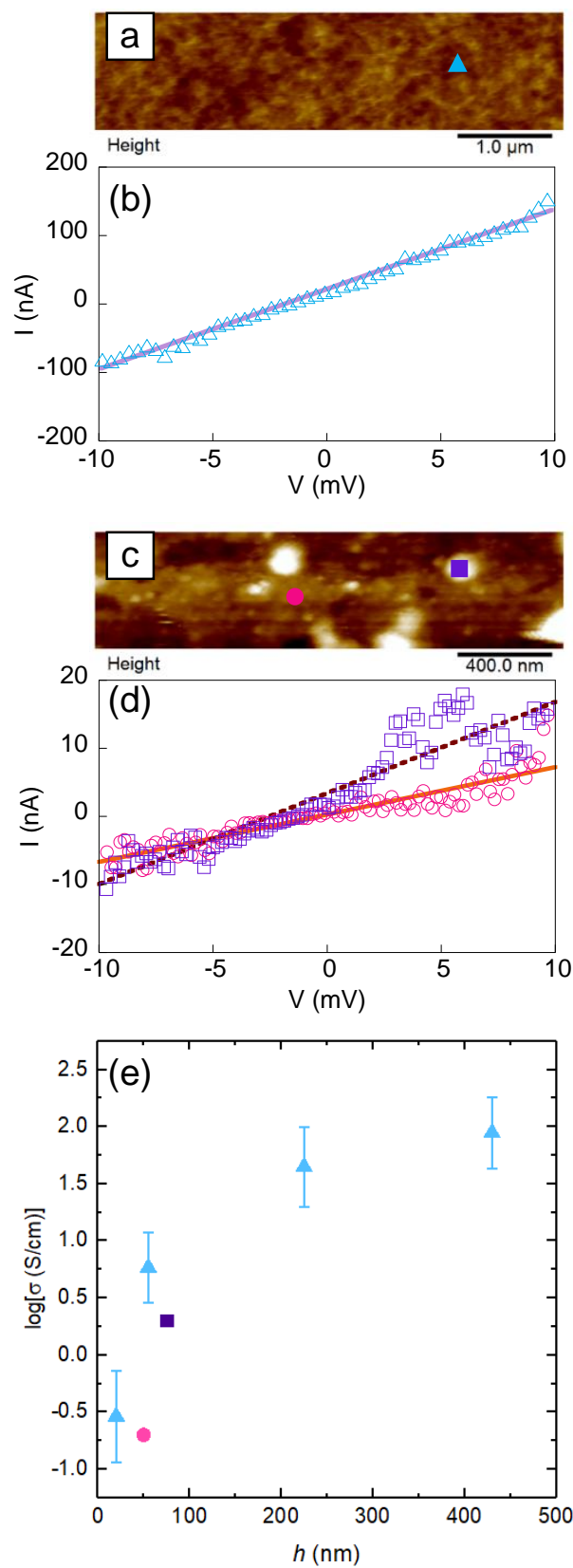

Figure 6. TUNA experiments on PEDOT:PSS thin film and nanospheres. (a) Sample 0 topography. (b) Sample $0 \mathrm{I}-\mathrm{V}$ curve at the position indicated in panel (a). The electric data is shown as 1 point every 10 measurements. The continuous line represents the linear fit from which the conductivity was calculated. (c) Sample 2 topography. (d) Sample $2 \mathrm{I}-\mathrm{V}$ curves at the points indicated in panel (c). The electric data is 
shown as 1 point every 20 measurements. The lines represent the linear fits for the circles (continuous line) and square (dashed line) data. (e) Logarithm of the conductivity as a function of thin film thickness (triangles). The values correspond to the mean over 5 measured points on different locations. The data shown as circle and square correspond to the conductivity at the probed zones of Sample 2

In order to understand how confinement effects might influence the PEDOT:PSS nanospheres electric transport, and to contextualize our results, we compared the nanospheres' conductivity values to those of PEDOT:PSS thin films with different thicknesses (Figure 6e). The TUNA measurements of these samples can be found in the Supplementary Information. We observed that as the film thickness decreased, so did the conductivity values. This result was in line with previous literature reports [58,59]. In this context, the prepared PEDOT:PSS nanospheres showed electric transport properties comparable to those for PEDOT:PSS thin films with thickness $<100 \mathrm{~nm}$. This result points out that the confinement effects on the nanospheres did not translate into a detriment of their conductivity properties.

Taking into consideration both the mechanical and electrical physical studies, we envisage that the prepared PEDOT:PSS nanospheres should have a PEDOT-rich shell, able to account for the stiffness reduction while preserving good electric transport properties. In this way, our investigation validates the fabrication of PEDOT:PSS nanospheres as a pathway to develop robust and electrical conducting nanostructures, via a single-step process.

\section{Conclusions}

A method to fabricate well-defined PEDOT:PSS nanospheres was developed, using a new strategy to solubilize PEDOT:PSS in the high boiling point solvent ethylene glycol. From the PEDOT:PSS solution, we fabricated the nanospheres in a single step using the reprecipitation methodology. The so obtained nanospheres' dispersions were characterized by DLS, which revealed that nanostructures of about $120 \mathrm{~nm}$ in diameter 
were prepared. The optical properties of the nanospheres were determined by UV-Vis spectroscopy, and resulted in features ascribable to PEDOT:PSS. This fact indicated that no important chemical modifications took during nanostructure fabrication. An in-depth AFM characterization on the dried nanospheres was carried out, able to quantify their structural, mechanical, and electrical properties. The nanoscale measurements indicated that the PEDOT:PSS nanospheres were able to preserve good mechanical properties, only showing a small decrease in their stiffness. The electrical measurements allowed to quantify the local conductivity of single nanospheres. The nanostructured PEDOT:PSS showed out-of-plane conductivity values in the 0.2 - $2 \mathrm{~S} / \mathrm{cm}$ range. These AFM physical studies suggested a different arrangement of PEDOT and PSS domains in the fabricated nanospheres respect to the commercial precursor, likely related to an enrichment of PEDOT onto the external shell of the nanospheres. Our results show the possibility of fabricating conducting polymer nanospheres, able to be integrated in future technologies.

\section{Conflict of interest}

The authors declare no conflict of interest.

\section{Acknowledgments}

A.A. acknowledges funding from Spanish Government "Ministerio de Ciencia, Innovacion y Universidades"' (PGC2018-094548-B-I00 (MCIU/AEI/FEDER, UE)), and funding by Basque Government (IT- 1175-19). D.E.M.-T. acknowledges the financial support obtained by the MCIU fellowship "Juan de la Cierva-Incorporación" (IJCI2017-31600). 


\section{References}

1. T K. Das, S. Prusty, Polymer-Plastics Technology and Engineering, 2012, 51, 1487.

2. X. Guo, A. Facchetti, Nature Materials, 2020, 19, 922.

3. S. Kirchmeyer, K. Reuter, J. Mater. Chem., 2005, 15, 2077.

4. Z. Fan, D. Du, H. Yao, J. Ouyang, ACS Appl. Mater. Interfaces, 2017, 9, 11732.

5. R. Singh, J. Tharion, S. Murugan, A. Kumar, ACS Appl. Mater. Interfaces, 2017, 9, 19427.

6. E. Vitoratos, S. Sakkopoulos, N. Paliatsas, K. Emmanouil, S. A. Choulis, Open Journal of Organic Polymer Materials, 2012, 2, 7.

7. L. Hu, J. Song, X. Yin, Z. Su, Z. Li, Polymers, 2020, 12, 145.

8. J. Song, G. Ma, F. Qin, L. Hu, B. Luo, T. Liu, X. Yin, Z. Su, Z. Zeng, Y. Jiang, G. Wang, Z. Li, Polymers, 2020, 12, 450.

9. J. Lee,Y. H. Kim, Synthetic Metals, 2018, 242, 99.

10. M. Beccatelli, M. Villani, F. Gentile, L. Bruno, D. Seletti, D. M. Nikolaidou, M. Culiolo, A. Zappettini, N. Coppedè, ACS Appl. Polym. Mater., 2021. In press.

11. U. Lang, E. Muller, N. Naujoks, J. Dual, Adv. Funct. Mater., 2009, 19, 1215.

12. T. Horii, H. Hikawa, M. Katsunuma, H. Okuzaki, Polymer, 2018, 140, 33.

13. H. J. Snaith, H. Kenrick, M. Chiesa, R. H. Friend, Polymer, 2005, 46, 2573.

14. L. Zhang, W. Du, A. Nautiyal, Z. Liu, X. Zhang, Sci. China Mater., 2018, 61, 3, 303.

15. J. Cárdenas-Martínez, B. L. España-Sánchez, R. Esparza, J. A. Ávila-Niño, Synthetic Metals, 2020, 267, 1164362.

16. J. H. Choi, H. J. Choi, J. H. Shin, H. P. Kim, J. Jang, H. Lee, Org. Electron., 2013, 14,3180 . 
17. C. W. Sele, T. v. Werne, R. H. Friend, H. Sirringhaus, Adv. Mater., 2005, 17, 8, 997.

18. E. Gutiérrez-Fernández, I. A. Gabaldón-Saucedo, Á. Rodríguez-Rodríguez, E. Solano, M. C. García-Gutiérrez, A. Nogales, A. Cirera, T. A. Ezquerra, E. Rebollar, Applied Surface Science, 2020, 509, 145350.

19. E. Jaworska, A. Michalska, K. Maksymiuk, Electroanalysis, 2017, 29, 123.

20. K. N. Schwarz, S. B. Farley, T. A. Smith, K. P. Ghiggino, Nanoscale, 2015, 7, 19899.

21. Z. Wang, J. Huang, W. Huang, H. Yamamoto, S. Kawaguchi, M. Nagai, Colloid and Polymer Science, 2019, 297, 69.

22. S. Zhang, J. Ren, Y. Zhang, H. Peng, S. Chen, F. Yang Y. Cao, Organic Electronics, 2020, 77, 105497.

23. C. H. Chen, A. Kine, R. D. Nelson, J. C. LaRu, Synthetic Metals, 2015, 206, 106.

24. C. Duc, G. Stoclet, J. Soulestin, C. Samuel, ACS Appl. Polym. Mater., 2020, 2, 2366.

25. B. Bessaire, M. Mathieu, V. Salles, T. Yeghoyan, C. Celle, J. P. Simonato, A. Brioude, ACS Appl. Mater. Interfaces, 2017, 9, 950.

26. J. Ouyang, C. W. Chu, F. C. Chen, Q.i Xu, Y. Yang, Adv. Funct. Mater., 2005, $15,2,203$.

27. H. Yan, H. Okuzaki, Synthetic Metals, 2009, 159, 2225.

28. K. Itoh, Y. Kato, Y. Honma, H. Masunaga, A. Fujiwara, S. Iguchi, T. Sasaki, J. Phys. Chem. C, 2019, 123, 13467.

29. O. P. Dimitriev, D.A. Grinko, Yu.V. Noskov, N.A. Ogurtsov, A.A. Pud, Synthetic Metals, 2009, 159, 2237. 
30. Y. H. Kim , C. Sachse, M. L. Machala, C. May, L. M. Meskamp, K. Leo, Adv. Funct. Mater., 2011, 21, 1076.

31. T. J. Young, M. A. Monclus, T. L. Burnett1, W. R. Broughton1, S. L. Ogin, P. A. Smith, Meas. Sci. Technol., 2011, 22, 125703.

32. R. Wagner, R.t Moon, J. Pratt, G. Shaw, and A. Raman, Nanotechnology, 2011, 22,455703 .

33. R. C. Germaniucus, D. Mercier, F. Agrebi, M. Febvre, D. Mariolle, Ph. Descamps, Ph, Leclere, Journal of Microscopy., 2020, 00, 0.

34. Sader J. E., Chon J. W. M., Mulvaney P., Rev. Sci.Instrum. , 1999, 70, 3967.

35. B. V. Derjaguin, V. M. Muller, Yu. P. Toporov, Journal of Colloid and Interface Science, 1975, 53, 2, 314 .

36. S. Fujinami, E. Ueda, K. Nakajima, T. Nishi, Journal of Polymer Science, B: Polymer Physics, 2019, 57, 1279.

37. U. Lang, N. Naujoks, J. Dual, Synthetic Metals, 2009, 159, 473.

38. Hans-Jürgen Butt, Brunero Cappella, Michael Kappl, Surface Science Reports, $2005,59,1$.

39. H. Yabu, Polymer Journal, 2013, 45, 261.

40. H. Shimizu, M. Yamada, R. Wada, M.Okabe, Polymer Journal, 2008, 40, 33.

41. W. Huang, C. Zhang, Biotechnol. J., 2018, 13, 1700203.

42. Edgar Gutiérrez-Fernández, Jing Cui, Daniel E Martínez-Tong, Aurora Nogales. Preparation, Physical Properties and Applications of Water-Based Functional Polymer Inks. In preparation. 2021.

43. E. Gutiérrez-Fernández, T. A. Ezquerra, E. Rebollar, Jing Cui, S. Marina, J. Martín, A. Nogales, Polymer, 2021, 218, 123515. 
44. S. Cheruku, L. D’Olieslaeger, N. Smisdom, J. Smits, D. Vanderzande, W. Maes, M. Ameloot, A. Ethirajan, Materials, 2019, 12, 2497.

45. L. D’Olieslaeger, Y. Braeken, S. Cheruku, J. Smits, M. Ameloot, D. Vanderzande, W. Maes, A. Ethirajan, Journal of Colloid and Interface Science, 2017, 504, 527.

46. J. De-La-Cuesta, I. Asenjo-Sanz, A. Latorre-Sánchez, E. González, D. E. Martínez-Tong, J. A. Pomposo, European Polymer Journal, 2018, 109, 447.

47. J. Wang, B. S. Fang, K. Y. Chou, C. C. Chen, Y. Gu, Enzyme and Microbial Technology, 2014, 54, 45.

48. H. W. Heuer, Rolf Wehrmann, and Stephan Kirchmeyer, Adv. Funct. Mater., $2002,12,2,89$.

49. P. Sakunpongpitiporn, K. Phasuksom, N. Paradee, A. Sirivat, RSC Adv., 2019, 9, 6363.

50. S. Khan and A. K. Narula, Eur. Polym. J., 2016, 81, 161.

51. Z. Z. You, Materials Letters, 2007, 61, 3809.

52. X. Crispin, S. Marciniak, W. Osikowicz, G. Zotti, A.W. Denier van der Gon, F. Louwet, M. Fahlman, L. Groenendaal, F.C. Schryver, de, W.R. Salaneck, Journal of Polymer Science, Part B: Polymer Physics, 2003, 41, 2561.

53. E. Gutiérrez-Fernández, I.A. Gabaldón-Saucedo, M.C. García-Gutiérrez, A. Varea, A. Nogales, E. Rebollar, A. Vilà, T.A. Ezquerra, A. Cirera, Organic Electronics, 2019, 70, 258.

54. D. Tahk, H. H. Lee, D. Y. Khang, Macromolecules, 2009, 42, 7079.

55. J. Qu, L. Ouyang, C. C. Kuo, D. C. Martin, Acta Biomater., 2016, 31, 114.

56. R. I. Rodríguez-Beltrán, Daniel E. Martínez-Tong, A, Reyes-Contreras, S. Paszkiewicz, A. Szymczyk, T.o A. Ezquerra, P. Moreno, E. Rebollar, Polymer, $2019,168,178$. 
57. R. C. Germanicus, D. Mercier, F. Agrebi, M. Febvure, D. Mariolle, Ph. Descamps, Ph. Leclere, Journal of Microscopy, 2020, 280, 1, 51.

58. M. Soccio, N. Lotti, A. Munari, E. Rebollar, D.E. Martínez-Tong, Polymer, 2020, $202,122666$.

59. A. J. Diaz, H. Noh, T. Meier, S. D. Solares, Beilstein J. Nanotechnol., 2017, 8, 2069.

60. E. S. Muckley, L. Collins, B. R. Srijanto, I. N. Ivanov, Adv. Funct. Mater., 2020, 30, 1908010.

61. F. Greco, A. Zucca, S. Taccola, A. Menciassi, T. Fujie, H. Haniuda, S. Takeoka, P. Darioab, V. Mattoli, Soft Matter, 2011, 7, 10642.

62. N. Saxena, M. C`oric', A. Greppmair, J. Wernecke, M. Pflüger, M. Krumrey, M. S. Brandt, E. M. Herzig, P. Müller-Buschbaum, Adv. Electron. Mater., 2017, 3, 1700181.

63. J. Liu, X. Wang, D. Li, N. E. Coates, R. A. Segalman, D. G. Cahill, Macromolecules, 2015, 48, 585.

64. Alexandre Mantovani Nardes, Martijn Kemerink, René A. J. Janssen, Jolanda A. M. Bastiaansen, Nicole M. M. Kiggen, Bea M. W. Langeveld, Albert J. J. M. van Breemen, and Margreet M. de Kok, Adv. Mater., 2007, 19, 1196. 


\section{Supplementary information file}

Figure S1. TUNA AFM results for PEDOT:PSS thin films at different thicknesses. Are reported respectively the topography image and the I-V curve of films with thickness: $430 \mathrm{~nm}(\mathrm{a}, \mathrm{b}), 225 \mathrm{~nm}(\mathrm{c}, \mathrm{d})$, $55 \mathrm{~nm}(\mathrm{e}, \mathrm{f})$, and $20 \mathrm{~nm}(\mathrm{~g}, \mathrm{~h})$.
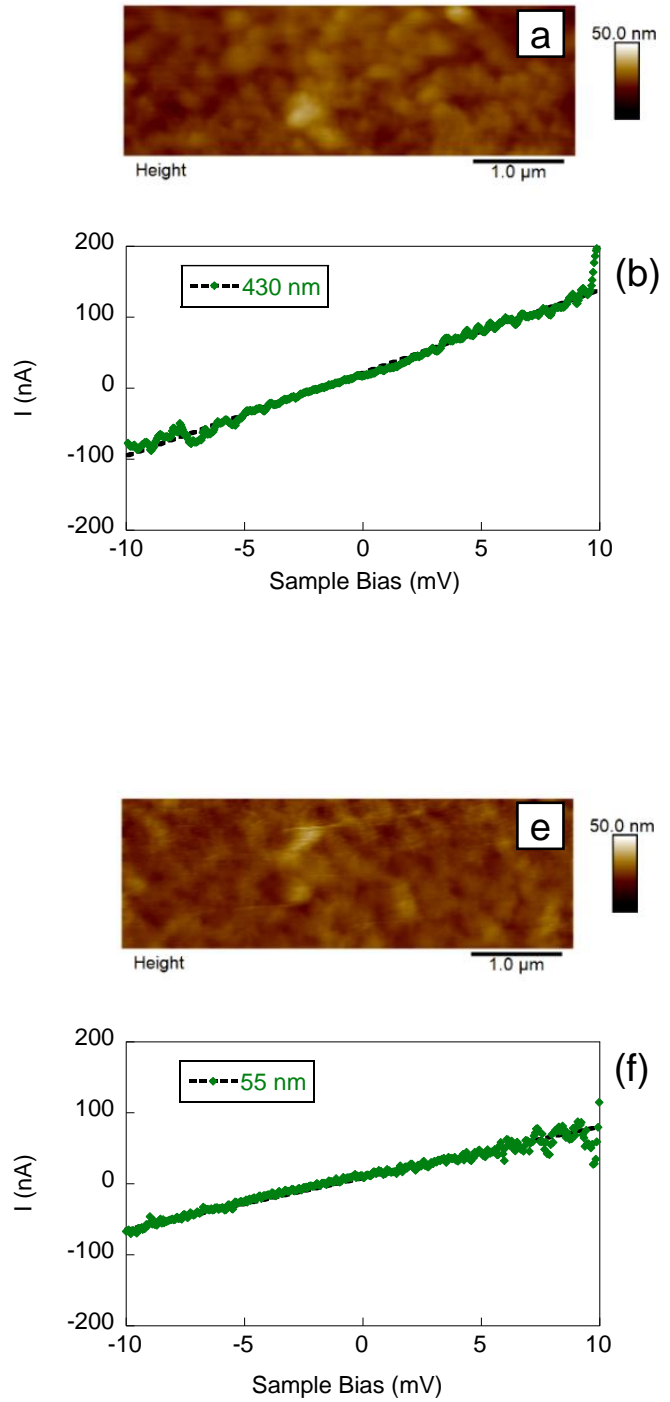
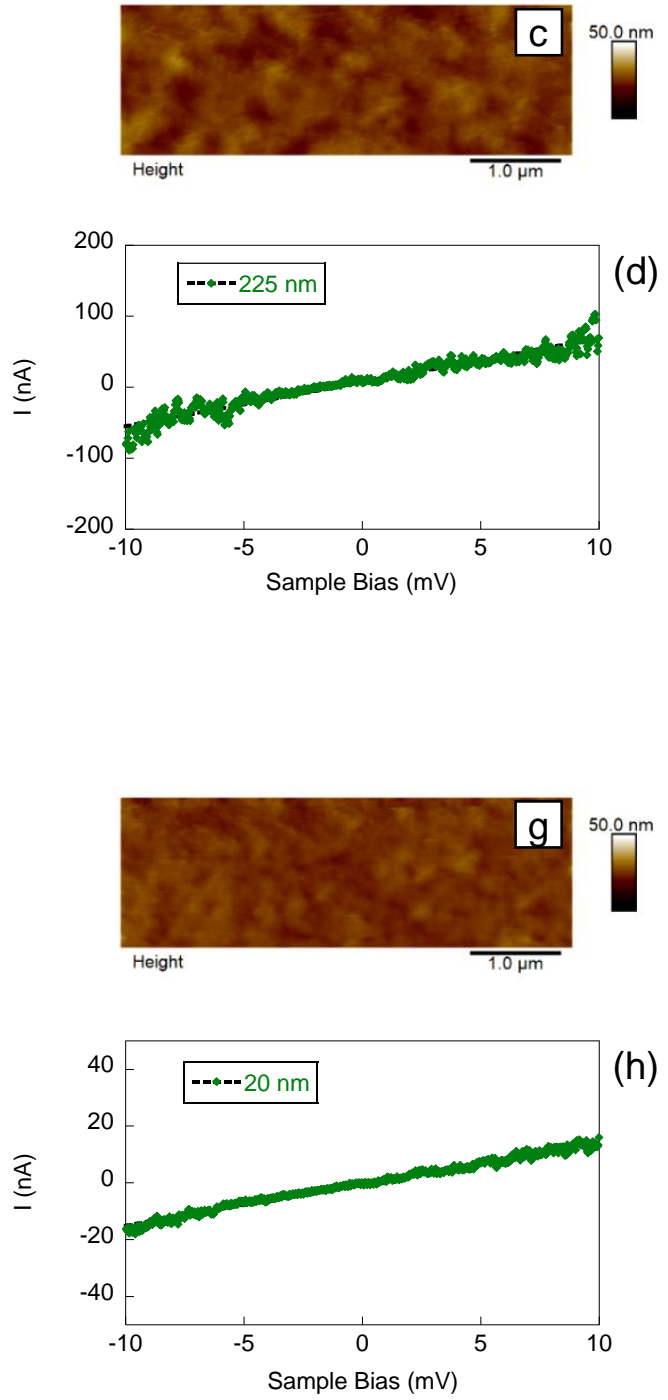

In Figure S1 it is reported the topography of PEDOT:PSS thin films with different thicknesses from Contact Mode TUNA AFM experiments. The I-V curves obtained in point-and shoot on the surface of the samples are also shown below each image. The topography of the PEDOT:PSS thin films, from the height channel, remains unchanged for all the considered thicknesses, while the slope of the lines reported in the graphs, representing the out-of-plane conductance of the films, showed a decrease as the thickness reduced. The quantitative values of conductivities can be found in the main manuscript. 